\title{
SERBIE
}

\section{INSTRUGTION SUR LA CONVENTION DE GENÈVE}

Ordonnance du Ministre de la guerre a tous les officiers, sous-officiers et soldats de l'armée régulière el de la milire.

Les lois de la guerre, en prenant sous leur protection spéciale les blessés et les malades militaires, étendent cette protection aux institutions qui ont pour but les soins à domner aux soldats malades et blessẹs en temps de guerre, ainsi qu'an personnel qui y est affeclé.

Les règles a observer ont été établies par la Convention de Genève du 22 aoùt 1864, à laquelle ont adhéré presque toutes les nations civilisées. Je crois done devoir faire connaître à toute l'armée les principes de la Convention de Genève, et j’enjoins à tous les officiers, sous-officiers et soldats, de se conformer strictement aux règles suivantes, en ce qui concerne les blessés et les malades des ambulances, lazarets et hopitaux militaires, aussi bien que ceux des hòpitaux créés pour la durée de la guerre par des sociétés ou personnes privées, et en ce qui concerne la position des blessés et des malades après leur guérison.

3. 1. Pendant toute la durée de la guerre, toutes les ambulances de campagne, d'étapes, de réserve, ainsi que tous les hôpitaux permanents et ceux établis par des sociétés particulières on des personnes privées, sont sous la protection des parties belligérantes et sont considérés comme établissements inviolables. Ils ne doivent être en butte à aucune hostilité. Il est interdit de tirer sur ces établissements, d'y mettre le feı, et en général de leur causer du dommage de quelque manière que ce soit. Comme tous les hôpitaux sont placés sans distinction sous la protection des parties belligérantes, il n'est besoin d'ancune force militaire pour faire respecter leur inviolabilité. Cependant, comme dans toute guerre il se présente des cas de maraudage, de vol, etc., il est nécessaire que chaque hòpital qui renferme des malades ou des blessés ait 
une garde, pour protéger le matériel de l'hòpilal et veiller à la tranquillité des blessés.

2. 2. Tontes les personnes attachées au service des ambulances et des hòpitaux, tant permanents que privés, telles que médecins, pharmaciens, commissaires, prètres, brancardiers, infirmiers, serviteurs sont inviolables pendant la durée de la guerre; elles jouissent de cette inviolabilité aussi longtemps qu'elles sont dans l'exercice de leur's fonctions, et aussi longtemps qu'il y a des malades ou des J)essés dans l'établissement auquel elles sont attachées. Ainsi les belligérants ont à respecter ces personnes, et ne peuvent leur donner un autre emploi que celui auquel elles étaient affectées. En général aucune violence ne doit être employée à leur égard. Elles ne peuvent pas être fátes prisonnières ni servir d'ôtages. Si leurs fonctions doivent cesser par la prise de la place où l'hòpital était établi, elles seront libres de retourner à leur armée.

3. 3. Le matériel des hòpitaux militaires, demeurant soumis aux lois de la guerre, peut être pris et gardé. Les personnes attachées à ces établissements ne peuvent emporter, en retournant à leur armée, que ce qui leur appartient en propre, mais rien de ce qui constitue le matériel de l'hòpital. Le matériel des ambulances et des lazarets de campagne, au contraire, ne peut être considéré comme butin. Le matériel des hôpitaux permanents, quoique de bonne prise, ne peut toutefois être considéré comme simple butin de guerre et être détourné de sa destination. Tous les lits, instruments, médicaments, objets de pansement, vêtements, etc., etc., doivent continuer à ètre affectés au traitement des malades et des blessés.

2. 4. Les habitants du pays ennemi qui porteront secours aux blessés devront être respectés et seront entièrement libres. Tout blessé recueilli dans une maison particulière y servira de sauvegarde. La maison sera dispensée du logement de troupes. L'habitant qui aura recueilli chez lui des blessés sera dispensé d'une partie des contributions de guerre imposées à la localité. On prendra en considération les soins qu'il aura donnés à des blessés on à des malades, ainsi que sa situation de fortune. Il va sans dire qu'il est parfaite. ment indifférent que les blessés ou les malades recueillis appartiennent à l'une ou à l'autre des parties belligérantes. Il ne peut être dirigé aucun acte d'hostilité contre une maison qui a recueilli des blessés ou des malades, à moins qu'il n'y ait nécessité militaire. 
Mais, dans ce dernier cas, des mesures doivent être prises à temps pour la mise en sûreté des blessés ou des malades. La personne qui a recueilli des blessés et s'est ainsi assurée certains priviléges les perdrait immédiatement, si l'on s'apercevait qu'elle en abuse, soit en cachant chez elle des espions ou des déserteurs, soit de quelque autre manière.

8. 5. Pendant et après le combat, les blessés et les malades sont relevés et soignés sans distinction de nationalité. Les commandants en chef peuvent remettre aux avant-postes ennemis les blessés et les malades ennemis, du consentement des deux parties et lorsque les circonstances le permettent. Si cela n'est pas possible, les blessés et malades ennemis seront recueillis dans nos ambulances. Ils recevront la même nourriture et les mêmes soins que nos propres blessés. Après guérison, ceux qui seront reconnus incapables de servir seront renvoyés dans leur pays. Ceux qui pourraient reprendre part à la guerre seront gardés dans notre pays, libres mais sous surveillance pendant la durée de la guerre. Celle-ci terminée ils seront renvoyés dans leur patrie. Les blessés enmemis sont par conséquent inviolables, non-seulement pendant qu'ils sont incapables de service mais encore après leur guérison. Tout ce qu'autoriseraient les lois de la guerre, ce serait de considérer comme prisonniers de guerre ceux qui, après leur guérison, pourraient de nouveau porter les armes.

8. 6. Comme signe distinctif pour assurer la neutralité des hópitaux et des ambulances militaires, ainsi que de toutes les personnes qui y sont attachées ou qui en général se consacrent au soulagement des blessés, la Convention de Genève a adopté pour les premiers le drapeau blanc à Croix rouge, et pour les dernières le brassard blanc à Croix rouge. Ce signe a été adopté par toutes les nations chrétiennes, signataires de la Convention de Genève; mais la Turquie a prié ces Etats, pour des motifs d'ordre religieux, de lui permettre de substituer à la Croix rouge sur fond blanc le Croissant rouge sur fond blanc. Les Etats signataires ont consenti à cette substitution, et la Serbie a déclaré, à la dale du 17 mai 1877 , qu'elle accorderait au drapeau blanc à Croissant rouge les mêmes égards qu'à la Croix rouge. Ainsi tous les bâtiments, tentes, etc., surmontés du drapeau blanc à Croissant rouge, de mème que toutes les personnes qui portent le brassard blanc à Croissant rouge, seront 
considérés comme neutres pendant la guerre, à l'égal de ceux qui seraient couverts par la Croix rouge. Aucuve hostilité ne doit être dirigée contre ces établissements ou ces personnes. Toutes les places de pansement doivent ètre aussi marquées par le drapeau blanc à Croix ou Croissant rouge, et l'on doit éviter de tirer dans leur direction, à moins qu'on ne puisse constater, d'une manière posilive, que l'ennemi abuse de ce signe. Si l'on reconnait que l'ennemi s'en sert pour couvrir des poudrières, des arsenaux, des dépôts de munitions, etc., il n'est plus opposé aux lois de la guerre de tirer sur ces bâtiments sans avoir égard an drapeau blanc. La responsabilité en retombe sur celui qui a commis l'abus, et non sur celni qui n'a pas tenu compte de l'inviolabilité du drapeau sanitaire, car il y a été amené par la mauvaise foi de l'adversaire

8. 7. La distribution des drapeaux blancs à Croix rouge, ainsique celle des brassards, a lieu sous le contròle sévère de l'autorité militaire, qui seule a le droit de les délivrer.

3. 8. Tout officier de l'armée serbe doit certifier par'sa signature qu'il a pris connaissance de la présente ordonnance.

Belgrade, le 1er décembre 1877.

Le Ministre de la guerre:

Sava Grouitch.

\section{NOUVEAUX STATUTS DE LA SOCIÉTÉ SERBE ${ }^{1}$}

Antiche 1er. - Du but et de l'ouvre de la Société serbe de la Croix rouge. La Société fondée sous le nom de Société serbe de la Croix rouge a pour but:

$1^{0}$ En temps de guerre: de concourir, à còté de l'administration sanitaire militaire de la principauté de Serbie et conformément aux ordres des autorités militaires, au soin des blessés et des malades de la guerre, et de les soutenir de toutes les manières possibles.

20 En temps de paix: de réaliser et réunir tout ce qui est nécessaire à ce concours en temps de guerre. L'oeuvre de la Société serbe de la Croix rouge consiste par conséquent :

1 Voir les anciens statuts, Bulletin, $n^{0} 27$, t. VII, p. 153. 